\title{
Food insecurity, weight control practices and body mass index in adolescents
}

\author{
Martin C Gulliford ${ }^{1, *}$, Cheryl Nunes ${ }^{2}$ and Brian Rocke ${ }^{2}$ \\ 'Department of Public Health Sciences, King's College London, Capital House, 42 Weston Street, London SE 1 3QD, \\ UK: ${ }^{2}$ Nutrition and Metabolism Division, Ministry of Health, Trinidad and Tobago
}

Submitted 8 June 2005: Accepted 25 October 2005

\begin{abstract}
Objective: We investigated whether experienced food insecurity was associated with weight control behaviour of adolescents.

Design: A national survey of 16-year-old students with the six-item food security scale, questions concerning intentions of trying to change weight, physical activity patterns, and measurement of height and weight.

Setting: Representative sample of 29 schools in Trinidad, West Indies.

Subjects: Data analysed for 1903 subjects including 1484 who were food-secure and 419 who were food-insecure.

Results: In the whole sample, food security status did not vary by body mass index (BMI) category. 'Trying to gain weight' and 'spending most free time in activities involving little physical effort' were each associated with lower BMI. 'Trying to gain weight' was more frequent in food-insecure subjects $(135,32 \%)$ than in food-secure subjects $(369,25 \%, P=0.012)$. After adjustment for BMI, age, sex, ethnicity and socioeconomic variables, the adjusted odds ratio (OR) of 'trying to gain weight' for food-insecure subjects was 1.39 ( $95 \%$ confidence interval (CI) $1.07-1.82, P=0.014$ ). Food-insecure subjects $(197,47 \%)$ were more likely than food-secure subjects $(575$, $39 \%$ ) to report that most of their free time was spent doing things that involved little physical effort $(P=0.003)$. This association was not explained by adjustment for BMI, age, sex and ethnicity (OR $=1.41,95 \%$ CI $1.13-1.76, P=0.002)$ or additional socioeconomic variables $(\mathrm{OR}=1.27,95 \%$ CI $1.02-1.57, P=0.033)$.

Conclusions: Adolescents who experience food insecurity are more likely to intend to gain weight but engage in less physical activity than food-secure subjects with the same BMI.
\end{abstract}

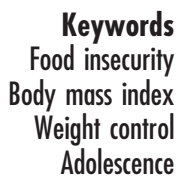

Food insecurity has been defined as the 'limited or uncertain availability of nutritionally adequate and safe foods, or limited or uncertain ability to acquire acceptable foods in socially acceptable ways', Experienced food insecurity is usually assessed through a standard questionnaire measure developed in the $\mathrm{USA}^{2}$. In the US Current Population Survey, some 12\% of all households, and between $36 \%$ and $42 \%$ of low-income households, were classified as food-insecure ${ }^{1}$. Surveys in London, England ${ }^{3}$ and Trinidad, West Indies ${ }^{4}$ have shown that food insecurity may be similarly or more frequent in some groups.

Research has suggested a possible association between food insufficiency or food insecurity and overweight which is best documented in adult women ${ }^{5-7}$. The association between food insecurity and overweight is generally observed in the context of moderate food insecurity, as more severe food insecurity may be associated with lower body mass index (BMI ${ }^{4}$. Several hypotheses have been advanced to explain why food insecurity might be associated with increased body weight ${ }^{8}$. First, food insecurity might lead to selection of obesity-promoting types of foods; second, food insecurity might lead to overeating when food is available; and third, food insecurity might lead to metabolic changes that result in more efficient utilisation of food. In support of the first of these hypotheses, several studies have shown that foodinsecure subjects may have diets which are considered less 'healthy', with lower intakes of fruit and vegetables and possibly higher intakes of energy-dense foods ${ }^{4,9,10}$. These patterns of food consumption might be explained by the food-insecure subjects' need to select low-cost foods ${ }^{11}$ and by the differential availability of foods to low-income groups 9 .

Connell et al. ${ }^{12}$ recently described the use of the US food security measure in older children and showed that it could be used successfully in this age group with minimal adaptation. Previously we evaluated the reliability and validity of the six-item version of the food security measure for use in adolescents, based on data from a 
sample in Trinidad, West Indies. We showed that item responses gave a satisfactory fit to the Rasch model ${ }^{10}$. Few studies have investigated possible associations between food insufficiency or food insecurity and body weight in adolescents. Alaimo et $a l^{8}$ evaluated whether food insufficiency was associated with overweight in children but found little evidence for an association except perhaps in older non-Hispanic white girls ${ }^{8}$.

If food insecurity is associated with the development of overweight, this association may be particularly relevant in adolescence when some health-related behaviours become established and problems of overweight and obesity may begin. The present report therefore extends our previous investigation of food insecurity in adolescents $^{10}$. We investigated whether experiences of food insecurity in adolescence are associated with either BMI or differences in behaviour in relation to body weight control. The study aimed to determine whether experienced food insecurity is associated with adolescents' perceptions of appropriate body weight, their intention to gain or lose weight, and their overall pattern of physical activity during leisure time.

\section{Methods}

\section{Sample}

A sample was drawn of children in Trinidad who had their 16 th birthday during the school year ${ }^{10}$. The sampling frame was a list of all 101 secondary schools in Trinidad. Schools were selected with probability proportional to size after stratifying by seven geographical administrative areas. Within schools, approximately 100 individual subjects were randomly selected from the school roll. The study received research ethics committee approval and was approved by the Ministries of Health and Education in Trinidad and Tobago. Subjects gave written informed consent to participate.

There were 30 schools sampled but one dropped out, leaving 29 schools with 3067 eligible students sampled for the survey. After excluding absent and refusing students there were 1973 (64\%) subjects with questionnaires and measurement forms returned. A further 70 subjects were excluded because of missing values for one or more of the six food security items. There were then 1903 (62\%) subjects available for analysis. There were four 'not known' responses to the weight perception question, three to the weight intention question and five to the physical activity question, and these were omitted. Six subjects had missing values for BMI.

\section{Data collection}

The questionnaire was self-completed by students in the classroom and fieldworkers answered any questions from the students. The questionnaire included the short-form, six-item household food security questionnaire as described by Blumberg et al. ${ }^{13}$. The performance of the measure in this adolescent sample has been reported ${ }^{10}$. Subjects were asked to complete two questions concerning their weight. The first concerned weight perceptions: 'Given your age and height, would you say that you are about the right weight, too heavy, too light, not sure?' The second question concerned weight intentions: 'At the present time, are you trying to lose weight, trying to gain weight or are you not trying to change your weight? A question was included concerning leisure-time physical activity: "Which one of the following statements describes you best?' Responses were: (1) 'All or most of my free time is spent doing things that involve little physical effort (e.g. watching TV, doing homework, talking to friends)'; (2) 'I occasionally (once or twice a week) do things in my free time that involve some physical effort (e.g. play sport, do running/jogging, cycling, aerobics or dancing)'; (3) 'I quite often (4-6 times a week) do things in my free time that involve some physical effort'; and (4) 'I very often (7 or more times per week) do things in my free time that involve some physical effort'. The questions concerning weight perceptions, weight intentions and physical activity were the same as those used in the Health Survey for England ${ }^{14}$. Measurements were made including height and weight following similar procedures to the Health Survey for England ${ }^{15}$. Subjects were measured in light clothing without shoes. Weight was measured using electronic digital scales (Soehnle) to the nearest $0.1 \mathrm{~kg}$. Height was measured using a portable stadiometer (Seca 'Leicester') to the nearest $0.1 \mathrm{~cm}$ below on the scale.

\section{Analysis}

The proportions of subjects who were overweight or obese were calculated using the proposed international standards $^{16}$ as well as the cut-off points of 25 and $30 \mathrm{~kg} \mathrm{~m}^{-2}$. Prevalence estimates are presented separately for the category of overweight or obese, referred to as 'overweight', and the category of 'obese'. BMI $Z$-scores were calculated from US BMI centiles ${ }^{17}$ using the Epi-Info package. Results were tabulated by BMI $Z$-score category. Odds ratios were estimated using a logistic regression model for multi-category data which was fitted using the MLOGIT command in Stata version $8^{18}$ using robust variance estimates to adjust for clustering by school. For analysis of the weight perception question, categories for 'about the right weight' and 'not sure' were combined and used for reference. For analysis of the weight intention question, the category of 'not trying to change weight' was used for reference. In order to make data presentation concerning physical activity more succinct, positive responses to the first option concerning little physical effort' were contrasted with all others in a logistic model. Initial analyses were adjusted for age (continuous variable), sex, ethnic group and BMI Z-score. Ethnicity was self-reported with categories reduced for analysis to 'African Caribbean', 'Indo-Caribbean', 'mixed' and 'other and not known'. In order to evaluate whether associations 
of food insecurity were independent of socio-economic position, analyses were additionally adjusted for type of drinking water supply in the home (piped in house, piped in yard, no piped supply), overcrowding quartile, number of parents in the home (both parents, father only, mother with male head of household, mother only, neither parent), paternal employment status (employed, unemployed, not known) and paternal education (none, primary, secondary, technical, university, not known), as in our previous report ${ }^{10}$.

\section{Results}

Based the international standard cut-off points for BMI, there were 135 (16.8\%) boys and 177 (16.1\%) girls who were overweight or obese and 55 (6.9\%) boys and 72 (6.5\%) girls who were obese. There were $13.7 \%$ of subjects who were overweight or obese using the BMI cut-off point of $25 \mathrm{~kg} \mathrm{~m}^{-2}$ and $5.9 \%$ using the cut-off point of $30 \mathrm{~kg} \mathrm{~m}^{-2}$.

Table 1 shows the distribution of responses to the weight perception and weight intention questions according to gender and BMI $Z$-score category. Both in boys and girls, perceptions of being 'too heavy' and intentions of 'trying to lose weight' increased with increasing BMI. At a given level of BMI, girls were more likely than boys to consider that they were too heavy and to be trying to lose weight. Perceptions of being 'too light' and 'trying to gain weight' were associated with lower BMI and decreased as BMI increased. Reporting that most free time was spent doing things that involved little physical activity was associated with lower BMI.

Food insecurity status was not associated with BMI category and tended to be most frequent at intermediate BMI (Table 1). The same finding was obtained with a more extended categorisation of BMI. In each category of BMI, subjects who experienced food insecurity were more often 'trying to gain weight' (Table 2). This association was not explained by adjusting for age, sex, ethnic group and socio-economic variables. The association was similar in boys and girls. Food insecurity was also associated with engaging in activities involving little physical effort (Table 2). This association was only slightly attenuated in the fully adjusted model. The association was slightly stronger in boys than girls. Food security status was not associated with subjects' weight perceptions or with intention to lose weight.

\section{Discussion}

Main findings and comparison with other studies

Comparison of the frequency of overweight and obesity between populations is difficult because of underlying systematic differences in height and weight-for-height between populations. International standards for

Table 1 Experienced food insecurity and perceptions of body weight and weight control practices according to BMI Z-score category for boys and girls

\begin{tabular}{|c|c|c|c|c|c|c|}
\hline & \multicolumn{3}{|c|}{ BMI Z-score } & \multirow[b]{2}{*}{ All* } & \multirow{2}{*}{$\begin{array}{c}\text { OR }(95 \% \mathrm{Cl}) \mathrm{per} \\
\text { unit change in BMI } \\
Z \text {-score† }\end{array}$} & \multirow[b]{2}{*}{$P$-value $\ddagger$} \\
\hline & $<-1$ & -1 to $<1$ & $\geq 1$ & & & \\
\hline Boys & 256 & 413 & 130 & 802 & & \\
\hline Food-insecure & $50(20)$ & $106(26)$ & $21(16)$ & $177(22)$ & $0.93(0.85-1.02)$ & 0.104 \\
\hline \multicolumn{7}{|l|}{ Weight perception } \\
\hline 'Too heavy' & $1(0)$ & $14(3)$ & $59(46)$ & $74(9)$ & $4.28(3.24-5.66)$ & $<0.001$ \\
\hline 'Too light' & $77(30)$ & $36(9)$ & $2(2)$ & $117(15)$ & $0.48(0.35-0.66)$ & $<0.001$ \\
\hline \multicolumn{7}{|l|}{ Weight intention } \\
\hline 'Trying to lose weight' & $8(3)$ & $72(17)$ & $97(75)$ & $178(22)$ & $3.74(2.93-4.76)$ & $<0.001$ \\
\hline 'Trying to gain weight' & $138(54)$ & 89 (22) & $7(5)$ & 236 (29) & $0.56(0.44-0.72)$ & $<0.001$ \\
\hline \multicolumn{7}{|l|}{ Physical activity } \\
\hline $\begin{array}{l}\text { 'Most free time spent doing things } \\
\text { involving little physical effort' }\end{array}$ & $79(31)$ & $105(26)$ & $32(25)$ & $217(27)$ & $0.89(0.78-1.01)$ & 0.073 \\
\hline Girls & 301 & 621 & 176 & 1101 & & \\
\hline Food-insecure & $58(19)$ & $143(23)$ & $39(22)$ & $242(22)$ & $1.00(0.87-1.14)$ & 0.990 \\
\hline \multicolumn{7}{|l|}{ Weight perception } \\
\hline 'Too heavy' & $2(1)$ & $86(14)$ & $94(54)$ & $182(17)$ & $3.86(3.12-4.78)$ & $<0.001$ \\
\hline 'Too light' & $82(27)$ & $35(6)$ & $2(1)$ & $120(11)$ & $0.41(0.32-0.52)$ & $<0.001$ \\
\hline \multicolumn{7}{|l|}{ Weight intention } \\
\hline 'Trying to lose weight' & $12(4)$ & $251(41)$ & $146(83)$ & 409 (37) & $4.15(3.24-5.30)$ & $<0.001$ \\
\hline 'Trying to gain weight' & $156(52)$ & 104 (17) & $5(3)$ & $268(24)$ & $0.44(0.35-0.56)$ & $<0.001$ \\
\hline \multicolumn{7}{|l|}{ Physical activity } \\
\hline $\begin{array}{l}\text { 'Most free time spent doing } \\
\text { things involving little physical effort' }\end{array}$ & $185(61)$ & $293(47)$ & $74(42)$ & $555(50)$ & $0.78(0.71-0.85)$ & $<0.001$ \\
\hline
\end{tabular}

$\mathrm{BMI}$ - body mass index; OR - odds ratio; $\mathrm{Cl}$ - confidence interval.

Figures are frequencies (percentage of column total).

* Three boys and three girls had missing values for BMI

$\dagger$ Adjusted for age and ethnic group.

$\ddagger$ Test for linear trend by BMI Z-score. 
Table 2 Proportion of subjects trying to gain weight by food security status, gender and BMI Z-score category

\begin{tabular}{|c|c|c|c|c|c|c|c|c|}
\hline & \multicolumn{3}{|c|}{ BMI Z-score } & \multirow[b]{2}{*}{ All ${ }^{*}$} & \multicolumn{2}{|c|}{ Model $1 \dagger$} & \multicolumn{2}{|c|}{ Model $2 \ddagger$} \\
\hline & $<-1$ & -1 to $<1$ & $\geq 1$ & & OR $(95 \% \mathrm{Cl}) \S$ & $P$-valuef & OR $(95 \% \mathrm{Cl}) \S$ & $P$-valueף \\
\hline \multicolumn{9}{|c|}{ ‘Trying to gain weight' } \\
\hline Food-secure & $228 / 449(51)$ & $128 / 782(16)$ & $10 / 246(4)$ & $369 / 1481(25)$ & - & _ & & \\
\hline Food-insecure & $66 / 108(61)$ & $65 / 249(26)$ & 2/60 (3) & $135 / 419(32)$ & $1.38(1.06-1.81)$ & 0.018 & $1.39(1.07-1.82)$ & 0.014 \\
\hline \multicolumn{9}{|c|}{ 'Most time spent doing things involving little physical effort' } \\
\hline Food-secure & $206 / 449(46)$ & $288 / 783(37)$ & $79 / 245(32)$ & $575 / 1481(39)$ & - & - & & \\
\hline Food-insecure & $58 / 108(54)$ & $110 / 247(45)$ & $27 / 60(45)$ & $197 / 417(47)$ & $1.41(1.13-1.76)$ & 0.002 & $1.27(1.02-1.59)$ & 0.030 \\
\hline
\end{tabular}

$\mathrm{BMI}$ - body mass index; OR - odds ratio; $\mathrm{Cl}$ - confidence interval.

Figures are frequencies (percentage of column total).

* Six subjects had missing BMI.

† Model 1: adjusted for age, sex and ethnic group and BMI Z-score.

¥ Model 2: adjusted for age, sex, ethnic group, BMI Z-score, water supply, parents at home, overcrowding, father's education and employment.

$\S$ Relative odds of trait if food-insecure compared with food-secure in adjusted model.

If Test for difference in frequency of trait comparing food-insecure and food-secure subjects in adjusted model.

overweight and obesity in children are recognised to be less applicable in populations of African or Indian subcontinent descent ${ }^{16}$. However, the overall frequency of overweight and obesity appeared to be lower in Trinidad and Tobago than in England, where 24\% of boys and $20 \%$ of girls were overweight or obese in $1997^{19}$. The prevalence of food insecurity in this sample was similar to the estimate of $25 \%$ from a household survey of adults in a more limited geographical area of $\operatorname{Trinidad}^{4}$, but higher than the overall prevalence of $12 \%$ estimated for the US population in the Current Population Survey ${ }^{1}$.

In adolescence, weight control practices are strongly associated with $\mathrm{BMI}^{14}$. Girls are more likely to report trying to lose weight but are less physically active than boys. After allowing for gender and BMI, we found that subjects who are food-insecure are more likely to be trying to gain weight and are less physically active than their food-secure counterparts. Weight control practices are associated with socio-economic position with higher socio-economic groups generally regulating against body weight gain more closely than lower socioeconomic groups ${ }^{20}$. Food insecurity is associated with lower socio-economic position ${ }^{3,4,9}$. However, the associations we describe are robust to adjustment for measures of socio-economic position which may be associated with food insecurity or weight regulation. The frequency of food insecurity varies by ethnic group $^{4}$ as does mean $\mathrm{BMI}^{21}$, but the associations described are also robust to adjustment for ethnicity and BMI. While the findings might provide indirect support to the hypothesis that food insecurity may lead to changes in body weight ${ }^{5,8}$, it was clear that there was no overall cross-sectional association between food insecurity and BMI in the present data. This raises questions concerning whether the self-reported variables are associated with changes in dietary intakes and energy expenditures that might contribute to weight changes, and whether sustained experience of food insecurity in this age group may lead to changes in body weight.

\section{Limitations of the study}

Our study was based on a representative sample of schools and gave a large sample for analysis. The response rate of $62 \%$ was comparable to that of other surveys. For example, in the Health Survey for England, $67 \%$ of subjects had their weight measured ${ }^{14}$. We used the short form of a well-described household food security measure ${ }^{13}$ which has been shown to be valid for use in Trinidad and Tobago $^{10,22}$. The food security items were designed to assess household food security. It has been shown that young children are generally protected from the consequences of household food insecurity until this is severe $^{23}$. However, the qualitative data of Connell et al. ${ }^{12}$, as well as our quantitative analyses ${ }^{10}$, show that older children are able to rate concerns of household food security. However, it may not always be the case that all individuals in food-insecure households experience the same consequences from food insecurity. The questions concerning weight perceptions, weight intentions and physical activity were the same as those used in the Health Survey for England ${ }^{14}$ with responses generally showing a similar distribution to those obtained for the English population. However, in Trinidad a higher proportion of girls responded that they were 'too light' and a higher proportion reported that they were trying to gain weight compared with English girls.

\section{Conclusions}

Experienced food insecurity may be associated with intention to gain weight and with low levels of leisure-time physical activity. These findings require confirmation in different settings and in different age groups. The observations raise a number of questions for future research. First, what are the cross-sectional associations between weight change intentions, or self-reported physical activity levels, with habitual energy intake and energy expenditure among adolescents? Second, if the experience of food insecurity and associated change in behaviour is sustained, is this associated with changes in 
body weight? Third, are interventions to address problems of food insecurity also associated with changes in weight control practices?

\section{Acknowledgements}

We thank the staff of the Central Statistical Office for help in drawing the sample and advice on conducting the survey, and the staff of the Nutrition Division for their skill and dedication in working on the survey. We acknowledge the key contribution of the late Dr Deepak Mahabir to the design and implementation of the study. We thank the journal's reviewers for comments contributing to the revision of the paper.

\section{References}

1 Carlson SJ, Andrews MS, Bickel GW. Measuring food insecurity and hunger in the United States: development of a national benchmark measure and prevalence estimates. Journal of Nutrition 1999; 129: 510S-6S.

2 Bickel G, Nord M, Price C, Hamilton W, Cook J. Measuring Food Security in the United States. Guide to Measuring Food Security, 2000 revision. Washington, DC: US Department of Agriculture, Food and Nutrition Service, 2000.

3 Tingay RS, Tan CJ, Tan NCW, Tang S, Teoh PF, Wong R, et al. Food insecurity and low income in an English inner city. Journal of Public Health Medicine 2003; 25: 156-9.

4 Gulliford MC, Mahabir D, Rocke B. Food insecurity, food choices, and body mass index in adults: nutrition transition in Trinidad and Tobago. International Journal of Epidemiology 2003; 32: 508-16.

5 Townsend MS, Peerson J, Love B, Achterberg C, Murphy SP. Food insecurity is positively related to overweight in women. Journal of Nutrition 2001; 131: 1738-45.

6 Adams EJ, Grummer-Strawn L, Chavez G. Food insecurity is associated with increased risk of obesity in California women. Journal of Nutrition 2003; 133: 1070-4.

7 Olson CM. Nutrition and health outcomes associated with food insecurity and hunger. Journal of Nutrition 1999; 129: $521 \mathrm{~S}-4 \mathrm{~S}$.

8 Alaimo K, Olson CM, Frongillo EA Jr. Low family income and food insufficiency in relation to overweight in US children: is there a paradox? Archives of Pediatrics \& Adolescent Medicine 2001; 155: 1161-7.
9 Tarasuk VS. Household food insecurity with hunger is associated with women's food intakes, health and household circumstances. Journal of Nutrition 2001; 131: 2670-6.

10 Gulliford MC, Mahabir D, Nunes C, Rocke B. Selfadministration of a food security scale by adolescents: item functioning, socio-economic position and food intakes. Public Health Nutrition 2005; 8: 853-60.

11 Aguirre P. Socio-anthropological aspects of obesity in poverty. In: Pena M, Bacallao J, eds. Obesity and Poverty: A New Public Health Challenge. Scientific Publication No. 576. Washington: DC: Pan-American Health Organization/World Health Organization, 2000; 11-22.

12 Connell CL, Nord M, Lofton KL, Yadrick K. Food security of older children can be assessed using a standardized survey instrument. Journal of Nutrition 2004; 134: 2566-72.

13 Blumberg SJ, Bialostosky K, Hamilton WL, Briefel RR. The effectiveness of a short form of the household food security scale. American Journal of Public Health 1999; 89: 1231-4.

14 Prescott-Clarke P, Primatesta P. Health Survey for England. The Health of Young People 1995-1997. London: The Stationery Office, 1999.

15 Colhoun H, Prescott-Clarke P. Health Survey for England 1994. London: HMSO, 1996.

16 Cole TJ, Bellizzi MC, Flegal KM, Dietz WH. Establishing a standard definition for child overweight and obesity worldwide: international survey. British Medical Journal 2000; 320: 1240-3.

17 Kuczmarski RJ, Ogden CL, Grummer-Strawn LM, Flegal KM, Guo SS, Wei R, et al. CDC growth charts: United States. Advance Data 2000; 8: 1-27.

18 Stata Corporation. Stata Reference Manual, Release 8. College Station, TX: Stata Corporation, 2001.

19 Jebb SA, Rennie KL, Cole TJ. Prevalence of overweight and obesity among young people in Great Britain. Public Health Nutrition 2004; 7: 461-5.

20 Wardle J, Griffith J. Socioeconomic status and weight control practices in British adults. Journal of Epidemiology and Community Health 2001; 55: 185-90.

21 Gulliford MC, Mahabir D, Rocke B, Chinn S, Rona RJ. Overweight, obesity and skinfold thicknesses of children of African or Indian descent in Trinidad and Tobago. International Journal of Epidemiology 2001; 30: 989-98.

22 Gulliford MC, Mahabir D, Rocke B. Reliability and validity of a short form household food security scale in a Caribbean community. BMC Public Health 2004; 4: 22.

23 Nord M, Bickel G. Measuring Children's Food Security in US Housebolds, 1995-99. Food Assistance and Nutrition Research Report No. 25. Washington, DC: Economic Research Service, US Department of Agriculture, 2002. 